\title{
Formation and Storage of on Demand Water-in-oil Picoliter Droplets Actuated by Microfluidic Pulse Inertia Force
}

\author{
Hongcheng Wang, Weiyi Zhang, Jia Liu, and Zhendong Dai
}

\begin{abstract}
Droplet generating in an immiscible liquid is the core technology of the droplet based biochemistry analysis. A new method, not based on micro-fluidic chip, is here proposed to produce micro scale aqueous phase droplets in oil phase liquid. The principle of the droplet generator is to actuate a hollow PZT stack by a signal generator and a voltage amplification to provide an enough pulse inertia force for a glass micro-nozzle and aqueous phase liquid inside to eject droplets. The minimum diameter of the produced spherical droplets is below $10 \mu \mathrm{m}$, which is below $1 \mathrm{pL}$. The range of droplets formation frequency is $2 \mathrm{~Hz} \sim 50 \mathrm{~Hz}$. A fused silica capillary is used to store the produced droplets by a designed low-pressure suction apparatus and the distance between the neighboring droplets is controlled by the low-pressure value. The proposed method in this article may be a promising approach for droplet-based single cell encapsulating and analyzing.
\end{abstract}

Index Terms-Microfluids, emulsions, pulse inertia force, picoliter droplets.

\section{INTRODUCTION}

Droplet-based microfluidic systems provide many new methods to perform reactions on a small scale [1]. Applications of these methods include chemical and biochemical screening, synthesis of particles [2], dynamic behavior analysis of single cells [3], directed evolution of enzymes [4], protein crystallization, polymerase chain reaction and analysis of gene expression [5] by encapsulating cells or different reagents in droplets with their volumes ranging from the nano- to femtoliter and the droplets usually is water-in-oil droplets, in which "water" is referred as dispersed phase while "oil" is referred as continuous phase. A variety of different droplet formation techniques have been developed, mainly including Channel-geometry-driven [6], Shear-flow-driven and Electro-driving. In shear-flow-driven droplet generation, the shearing force of one flowing fluid against another is used to form droplets. Three main types of shear-flow-driven droplet generation exist: T-junction [7],

Manuscript received September 5, 2014; revised March 30, 2015. This work was supported by Postdoctoral Science Foundation of China (no. 2014M551584) and National Natural Science Foundation of China (no. 51175268, no. 11102090).

Hongcheng Wang and Zhendong Dai are with the Institute of Bio-inspired Structure and Surface Engineering, Nanjing University of Aeronautics and Astromautics, Nanjing, CO 210016, China (e-mail: whch31@163.com,zddai@nuaa.edu.cn).

Weiyi Zhang and Jia Liu are with the School of Mechanical Engineering, Nanjing University of Science and Technology, Nanjing, CO 210094, China (e-mail: zhangweiyi_email@163.com,njustliujia@gmail.com). flow focusing [8] and Co-flowing [9]. In the above three shear-flow-driven droplet generators, the fluids are usually driven by syringe pumps and the sizes of the droplets can be changed by altering the fluid flow rates, the channel widths, or by changing the relative viscosity between the two phases. In electro-driven droplet generation [10], [11], electrodes are integrated into microdevices to provide electronical control over droplet formation. The great majority of the above microfluidic devices, as found in the literature, are based on micro-fluidic chip which is fabricated by standard soft lithography technique.

Different from all of the above types of droplet formation techniques, this work is to develop a new method to generate and store water-in-oil picoliter droplets actuated by microfluidic pulse inertia force based on Technology of Micro-Fluidics Digitalization [12].

\section{EXPERIMENT}

As is shown in Fig. 1, an apparatus for dispensing and storing aqueous phase droplets in an oil phase liquid based on microfluidic pulse inertia force was established. The micro-nozzle is clamped by a nozzle holder, which is fixed with the right face of the hollow PZT stack actuator (WTYDCR, CETC) through connector A while the left face is fixed with a three-dimension adjusting frame and kept stationary through connector B. The hollow PZT stack actuator is constructed of several disc-shaped piezoelectric ceramic pieces, the thickness of which is in the range of 0.02 $\mathrm{mm}$ to $1 \mathrm{~mm}$. There is an approximate linearity between applied voltage amplitude and the right face displacement of the actuator. So, the actuator will cause a larger displacement instantaneously and consequently provide a greater pulse inertia force for the nozzle and aqueous phase liquid inside when being applied a higher pulse driving voltage.

Glass material was chosed to make the micro-nozzle because of several advantages, such as good chemical resistance, low friction, easy to manufacture and low cost. The raw material is borosilicate glass capillary (Beijing Zhengtianyi Scientific And Trading Co., Ltd.). The dimensions of glass capillary are $1.6 \mathrm{~mm}, 1.0 \mathrm{~mm}$ and 100 $\mathrm{mm}$ in external diameter, internal diameter and length, respectively. Glass heating process was adopted to fabricate the micro-nozzle without complicated microfabrication technologyd can be divided into two steps: 1) pulling a capillary to form a micro-nozzle with straight outlet and 2) forging the straight outlet to form a shrinkage one. The detail fabrication process for the micro-nozzle was presented in literature [13]. The micro-nozzles with different outlet 
diameters can be obtained by varying the control parameters of the voltage amplitude and the balance weights. The minimum outlet diameter of the fabricated micro-nozzle can reach as small as $10 \mu \mathrm{m}$. The fabricated micro-nozzles with different outlet diameters are shown in Fig. 2.

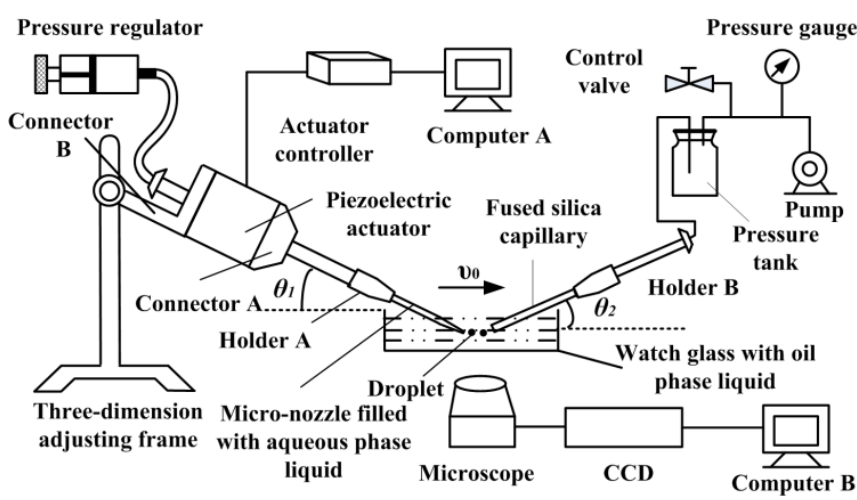

Fig. 1. Apparatus for dispensing and storing aqueous phase droplets in an oil phase liquid.

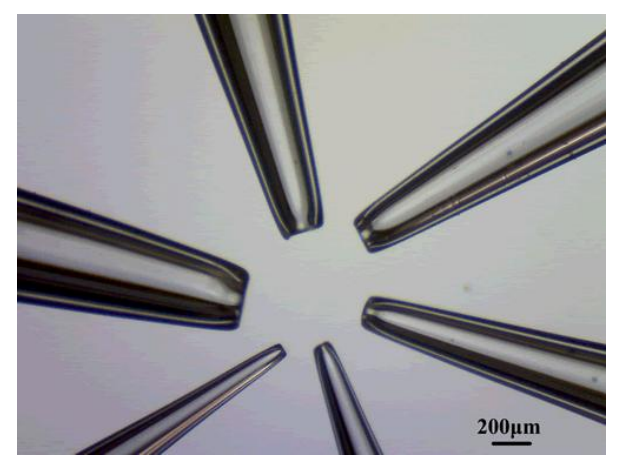

Fig. 2. Micrograph of the micro-nozzles with different outlet diameters.

The micro-nozzle inhaled a certain amount of aqueous phase liquid by capillary action or small negative pressure in the micro-nozzle produced by a pressure regulator. The micro-nozzle orifice is immersed in the oil phase liquid in a watch glass, which is placed on the stage of a chatelier-type microscope (TS-100, Nikon). A fused silica capillary (China Reafine Chromatography LTD.) clamped by holder B is used to store the generated droplets. As is shown in Fig. 3, the fused silica capillary is interconnected with a pressure tank through a PTFE tube. The low air pressure is produced by an air pump and precisely controlled by a pressure gauge.

The inner surface of the fused silica capillaries is hydrophobized with aqueous solution of $1 \%$ fluoroalkyl silane to prevent the generated droplets from adhering to the capillaries. The following procedures are experimentally feasible. Firstly, wash the fused silica capillaries with alcohol and dry them in an electric oven (DHG, Shanghai Jingmai Instruments Equipment Co., LTD). The temperature of $100^{\circ} \mathrm{C}$ was s adopted in the experiment and the temperature holding time is $20 \mathrm{~min}$. Secondly, filled the capillaries with aqueous solution of $1 \%$ fluoroalkyl silane using a syringe and dry them in the oven twice. The temperatures of the two drying process were set as $50^{\circ} \mathrm{C}$ and $110^{\circ} \mathrm{C}$ respectively with the same holding time of $20 \mathrm{~min}$. The lower temperature of $50^{\circ} \mathrm{C}$ was firstly adopted to prevent forming fluoroalkyl silane bulge films in the inner surface of the fused silica capillaries.
In the experiment, deionized water was used as aqueous phase liquid and mineral oil (M5904, American Sigma-Aldrich Company) was used as oil phase liquid. The mineral oil was added with $10 \%$ span 80 , a kind of surfactant, to prevent the generated deionized water droplets from being fused.

\section{RESULTS AND DISCUSSION}

As shown in Fig. 4, the deionized water droplets were ejected from the micro-nozzle with outlet diameter of $30 \mu \mathrm{m}$ by microfluidic pulse inertia force in the mineral oil environment. A droplet will be produced in a pulse driving period. In other words, the droplet formation frequency is equal to the frequency of driving signal. The generated droplets were inhaled into the fused silica capillary successively at equal distance by the low air pressure in it produced by the low-pressure suction apparatus recorded by the chatelier-type microscope (TS-100, Nikon) and the CCD camera (WV-CP230/G, Panasonic). The distance between the neighboring droplets can be controlled by varying value of negative pressure. The size of the droplets, which is characterized by the diameter of the spherical shape, can be controlled by the pulse inertia force, which is determined by outlet diameter of the micro-nozzle and shape, frequency, amplitude of the applied pulse voltage on the hollow PZT stack actuator.

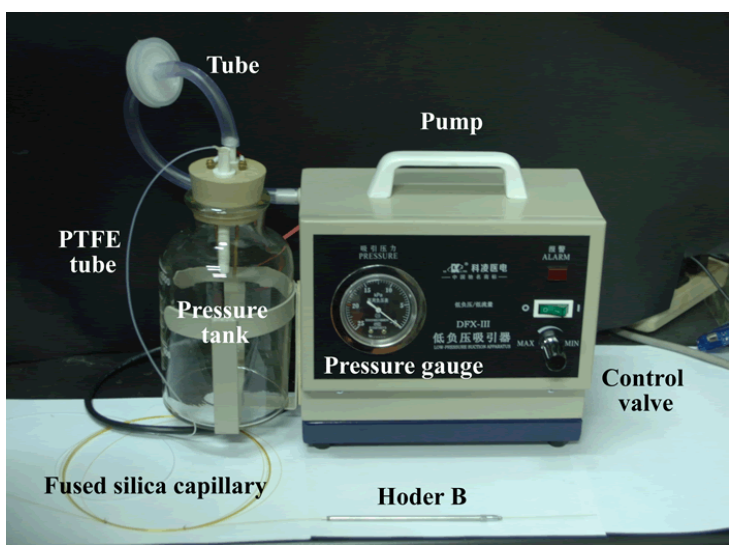

Fig. 3. Photo of low-pressure suction apparatus for storing generated droplets.

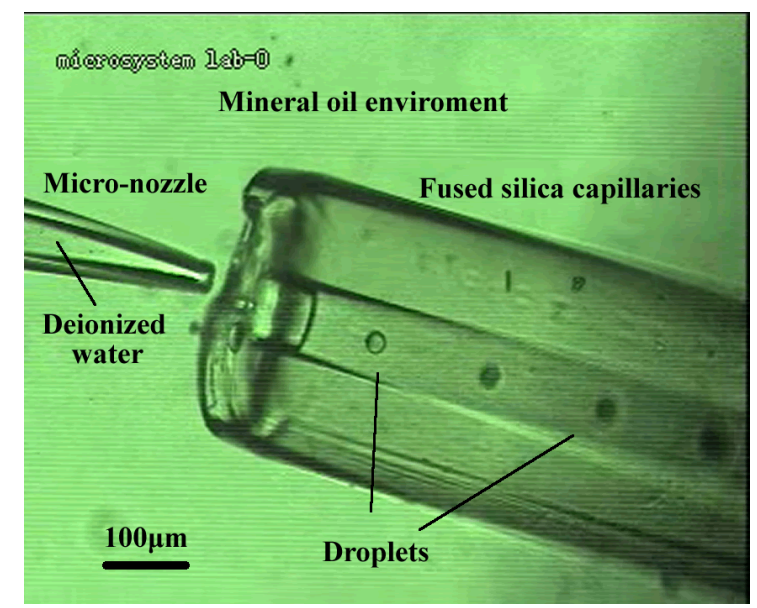

Fig. 4. Jetting process of deionized water droplets from a micro-nozzle in mineral oil 
The dependence of deionized water droplet size on voltage amplitude for different diameters of nozzle outlet is shown in Fig. 5. The frequency of the applied voltage is set as a lower value of $2 \mathrm{~Hz}$. Each data point is an average of ten measurements. The hollow PZT stack actuator causes a larger displacement instantaneously and consequently provides greater pulse inertia force for the micro-nozzle and aqueous phase liquid inside when being applied a higher driving voltage. So, deionized water droplets will be ejected and the droplet size increased with the increase of voltage amplitude. If the voltage is lower in magnitude, deionized water droplets won't be ejected when the micro-nozzle size is relatively small. For instance, the minimum voltage to eject droplets is $30 \mathrm{~V}$ and $20 \mathrm{~V}$ for nozzle orifice diameters of $10 \mu \mathrm{m}$ and 20 $\mu \mathrm{m}$ respectively. On the other hand, when the voltage amplitude is relatively high, the inertia force was big enough to eject more mass of aqueous phase liquid, thus forming satellite droplets. For instance, the maximum voltage to stably eject droplets without satellite droplets is $50 \mathrm{~V}$ when the nozzle orifice diameter is $60 \mu \mathrm{m}$. The satellite droplets are shown in Fig. 6.

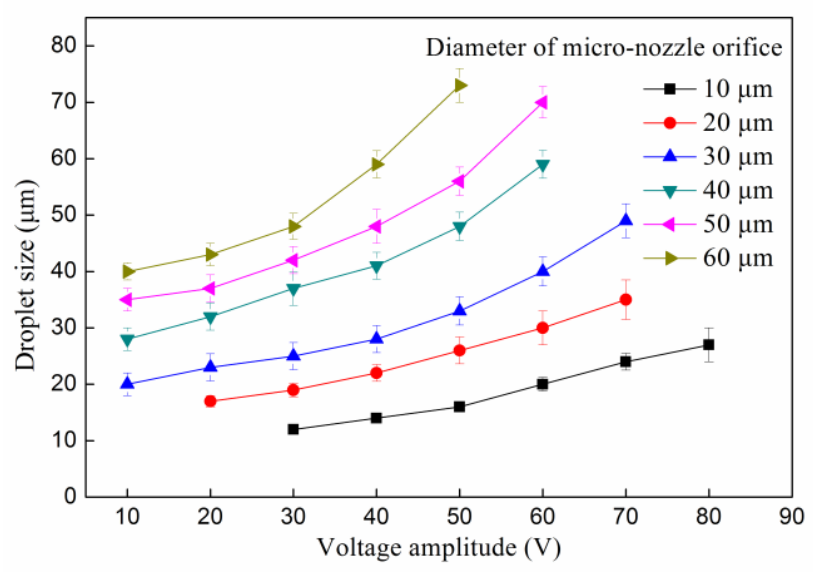

Fig. 5. Variation of the droplet size with voltage amplitude and micro-nozzle outlet diameters.

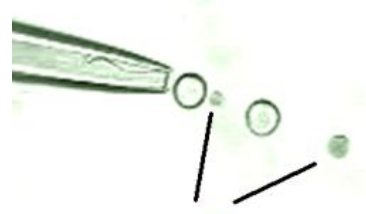

Satellite Droplets

$100 \mu \mathrm{m}$

Fig. 6. Micrograph of deionized water droplets with satellite droplets.

As is shown in Fig. 7, the droplet size decreased with the increase of the voltage frequency under different voltages. However, when the voltage frequency is above $30 \mathrm{~Hz}$, the droplets cannot be generated with the voltage amplitudes of $70 \mathrm{~V}$. The reason is that it needs time for PZT stack actuator to response to the driving voltage signal. If the voltage amplitude is too high, the actuator will produce a larger displacement and the response time is relatively long, which will cause interference between the neighboring driving voltage periods and the produced pulse inertia force is not big enough to form droplets.

The average diameter of these spherical droplets in Fig. 4 is about $25 \mu \mathrm{m}$ and their shape and size have a good consistent in the condition of outlet diameter of the micro-nozzle $30 \mu \mathrm{m}$, voltage frequency $2 \mathrm{~Hz}$, voltage amplitude $30 \mathrm{~V}$ and negative pressure value $5 \mathrm{kPa}$. The distance between the neighboring droplets is about $160 \mu \mathrm{m}$. The droplets with different sizes and different distances between the neighboring droplets are stored in silica capillaries, as is shown in Fig. 8. The droplet sizes are $10 \mu \mathrm{m}$, $10 \mu \mathrm{m}, 20 \mu \mathrm{m}$ and $50 \mu \mathrm{m}$ and average distances between the neighboring droplets are $100 \mu \mathrm{m}, 80 \mu \mathrm{m}, 30 \mu \mathrm{m}$ and $100 \mu \mathrm{m}$ respectively.

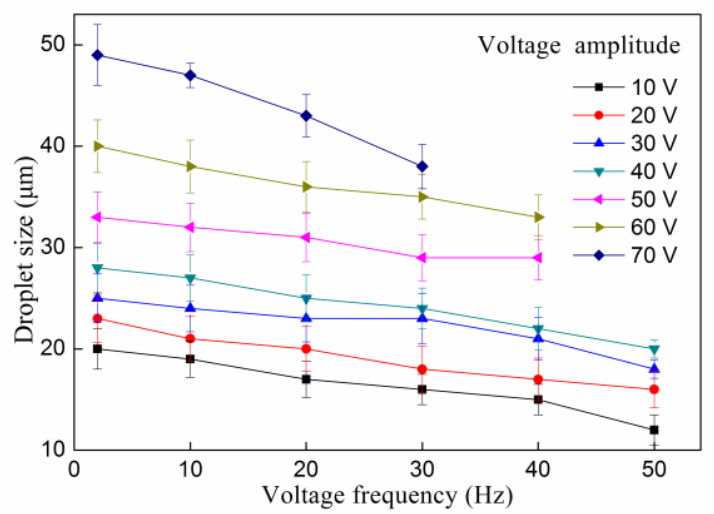

Fig. 7. Variation of the droplet size with voltage frequency and voltage amplitude.

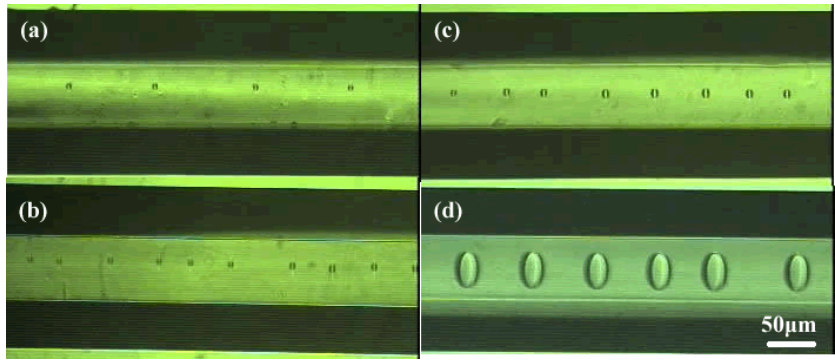

Fig. 8. Micrograph of the droplets stored in fused silica capillaries with different sizes and different average droplet distances.

\section{CONCLUSION}

We have presented a new method of producing micro scale aqueous phase droplets in oil phase liquid actuated by microfluidic pulse inertia force and the generated droplets are stored in a fused silica capillary by a designed low-pressure suction apparatus. In the process of the droplet formation, the droplet size can be easily changed by changing any one of the micro-nozzle outlet diameter and the shape, frequency, amplitude of the applied pulse voltage. The minimum diameter of these produced spherical droplets is below $10 \mu \mathrm{m}$ and the maximum diameter is above $70 \mu \mathrm{m}$ as well. The droplet formation frequency is equal to the frequency of driving signal applied on the PZT stack actuator and should be set below $50 \mathrm{~Hz}$ to avoid satellite droplets. The experiment result suggests that our method may be applied in droplet-based single cell encapsulating and analyzing. 


\section{ACKNOWLEDGMENT}

The work was supported by Postdoctoral Science Foundation of China (no. 2014M551584) and National Natural Science Foundation of China (no. 51175268, no. 11102090).

\section{REFERENCES}

[1] H. Song and D. L. Chen, "Reactions in droplets in microfluidic channels," Ismagilov: Angew. Chem. Int. Ed., vol. 45, pp. 7336-7356, Nov. 2006.

[2] T. D. Dang, Y. H. Kim, H. G. Kim, and G. M. Kim, "Preparation of monodisperse PEG hydrogel microparticles using a microfluidic flow-focusing device," J. Ind. Eng. Chem., vol. 18, pp. 1308-1313, Jan. 2012.

[3] M. A. Khorshidi, P. K. P. Rajeswari, C. Wähilby, H. N. Joensson, and H. A. Svahn "Automated analysis of dynamic behavior of single cells in picoliter droplets," Lab Chip, vol. 14, pp. 931-937, 2014.

[4] S. L. Sjostrom, Y. P. Bai, M. T. Huang, Z. H. Liu, J. Nielsen, H. N. Joensson, and H. A. Svahn, "High-throughput screening for industrial enzyme production hosts by droplet microfluidics," Lab Chip, vol. 14, pp. 806-813, 2014.

[5] D. Y. Liu, G. T. Liang, X. X. Lei, B. Chen, W. Wang, and X. M. Zhou, "Highly efficient capillary polymerase chain reaction using an oscillation droplet microreactor," Anal. Chim. Acta., vol. 718, pp. 58-63, 2012.

[6] M. Nagel, P. T. Brun, F. Gallaire, "A numerical study of droplet trapping in microfluidic devices," Phys. Fluids, vol. 26, pp. 032002, 2014.

[7] Y. Shi, G. H. Tang, and H. H. Xia, "Lattice Boltzmann simulation of droplet formation in T-junction and flow focusing devices," Computers \& Fluids, vol. 90, pp. 155-163, 2014.

[8] A. Gupta, H. S. Matharoo, D. Makkar, and R. Kumar, "Droplet formation via squeezing mechanism in a microfluidic flow-Focusing device," Computers \& Fluids, vol. 100, pp. 218-226, 2014.

[9] C. Cramer, P. Fischer, and E. J. Windhab, "Drop formation in a co-fowing ambient fuid," Chem. Eng. Sci., vol. 59, pp. 3045-3058, 2004.

[10] T. B. Jones, M. Gunji, M. Washizu, and M. J. Feldman, "Dielectrophoretic liquid actuation and nanodroplet formation," $J$. Appl. Phys., vol. 89, pp. 1441-1448, 2001.

[11] Y. Y. Lin, E. R. F. Welch, and R. B. Fair, "Low voltage picoliter droplet manipulation utilizing electrowetting-on-dielectric platforms," Sens. Actuators B, vol. 173, pp. 338-345, 2012.

[12] W. Y. Zhang and L. Y. Hou, "Method, apparatus and application of affecting fluid flow," China Patent ZL03152948. 8, 2006.
[13] H. C. Wang, L. Y. Hou, and W. Y. Zhang, "A drop-on-demand droplet generator for coating catalytic materials onmicrohotplates of micropellistor," Sens. Actuators B, vol. 183, pp. 342-349, 2013.

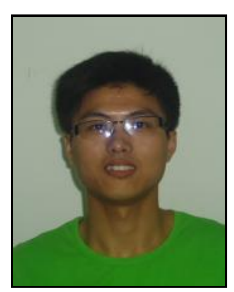

Hongcheng Wang received his B.E degree in 2008 from Nanjing Institute of Technology at Nanjing, Jiangsu, China. He received his Ph.D. degree in School of Mechanical Engineering, Nanjing University of Science and Technology (NUST), Nanjing, China, in 2013. Now he is a postdoctor in Institute of Mechanical and Electrical Engineering of Nanjing University of Aeronautics and Astromautics (NUAA). His research interests include microfluidic system, microfabrication technology and biochemical engineering.

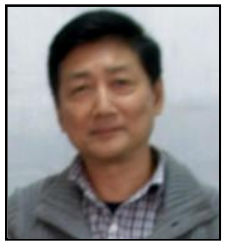

Weiyi Zhang received a Ph.D. degree from Beijing University of Aeronautics \& Astronautics at Beijing, China. He worked as a visiting fellow from 1992 to 1995 in Hosei University at Tokyo, Japan. Since 1996, she has worded at NUST as a professor and founder of Microsystem Laboratory. He has focused on microfluidic system, microsystem and nanosystem, digitalization of microfluids technology and micro robotics for biochemical engineering.

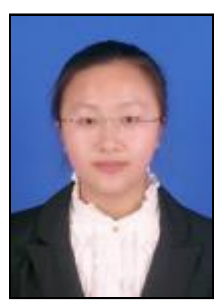

Jia Liu received her BE degree in mechanical engineering from NUST in 2013. Currently, she is a $\mathrm{PhD}$ course student at Graduate School of Engineering, NUST. Her research interest is in microfluidics.

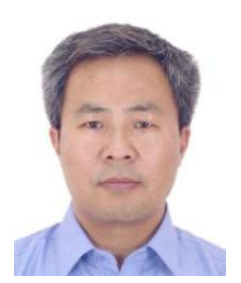

Zhendong Dai received his Ph.D. degree from Institute of Mechanical and Electrical Engineering of NUAA, Nanjing, China in 2000. Since 2001, he has worded at NUAA as a professor and now he is the chief of the Institute of Bio-inspired Structure and Surface Engineering. His research interest is structure of bionics, surface engineering and biological science. 